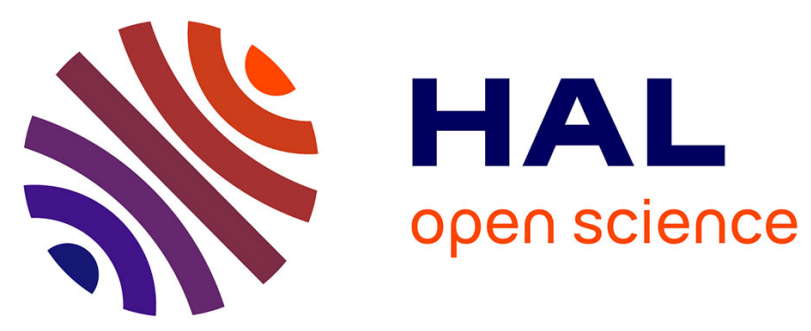

\title{
Feasibility of fast MR-thermometry during cardiac radiofrequency ablation
}

\author{
Baudouin Denis de Senneville, Sébastien Roujol, Pierre Jaïs, Chrit T. W. \\ Moonen, Gwenaël Herigault, Bruno Quesson
}

\section{- To cite this version:}

Baudouin Denis de Senneville, Sébastien Roujol, Pierre Jaïs, Chrit T. W. Moonen, Gwenaël Herigault, et al.. Feasibility of fast MR-thermometry during cardiac radiofrequency ablation. NMR in Biomedicine, 2012, 25 (4), pp.556-562. 10.1002/nbm.1771 . hal-01578212

\section{HAL Id: hal-01578212 \\ https://hal.science/hal-01578212}

Submitted on 29 Aug 2017

HAL is a multi-disciplinary open access archive for the deposit and dissemination of scientific research documents, whether they are published or not. The documents may come from teaching and research institutions in France or abroad, or from public or private research centers.
L'archive ouverte pluridisciplinaire HAL, est destinée au dépôt et à la diffusion de documents scientifiques de niveau recherche, publiés ou non, émanant des établissements d'enseignement et de recherche français ou étrangers, des laboratoires publics ou privés. 


\section{Feasibility of fast MR-thermometry during cardiac RF ablation}

Baudouin Denis de Senneville, Sébastien Roujol, Pierre Jaïs, Chrit T. W. Moonen, Gwenaël Herigault, Bruno Quesson
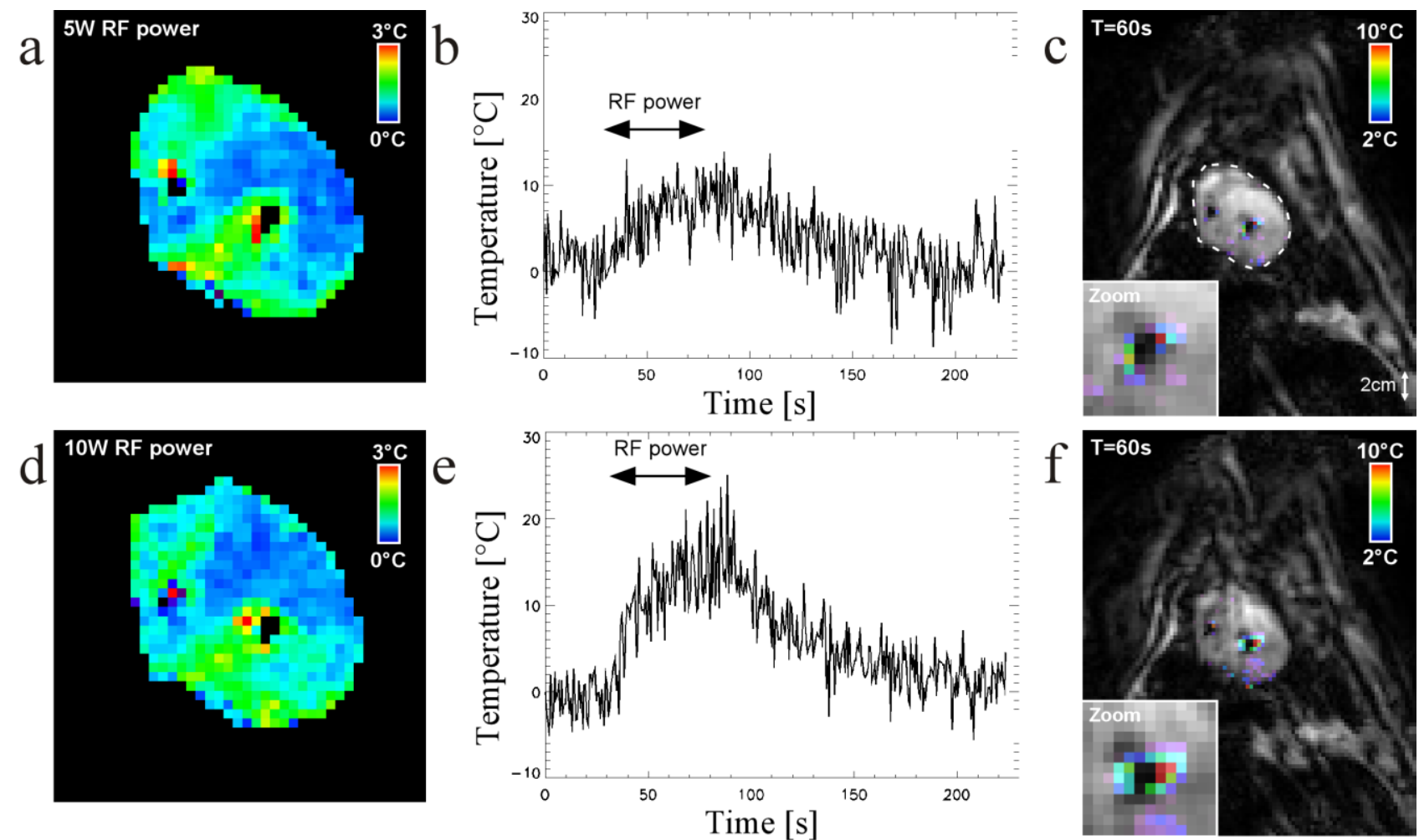

Online MR temperature monitoring during radiofrequency (RF) ablation of cardiac arrhythmias may improve the efficacy and safety of the treatment. MR thermometry at 1.5 Tesla with the Proton Resonance Frequency method was assessed in 10 healthy volunteers under normal breathing conditions, using a multi-slice, ECG-gated, Echo Planar Imaging sequence in combination with slice tracking. Temperature images were post-processed to remove residual motion-related artifacts. Using an MR-compatible steerable catheter and electromagnetic noise filter, RF ablation was performed in the ventricles of sheep in vivo. Tissue temperature could be monitored at an update rate of approximately 1 $\mathrm{Hz}$ in five slices. Typical temperature changes observed during clinical RF application could also be monitored with an acceptable level of precision. 


\title{
Feasibility of fast MR-thermometry during cardiac RF ablation
}

\author{
Baudouin Denis de Senneville, $\mathrm{PhD}^{1}$, Sébastien Roujol, M.Sc. ${ }^{1}$, Pierre Jaïs ${ }^{1,2}$, MD ${ }^{1}$, Chrit T. W. \\ Moonen, PhD ${ }^{1}$, Gwenaël Herigault, M.Sc. ${ }^{3}$, Bruno Quesson, PhD ${ }^{1}$
}

1: Laboratory for Molecular and Functional Imaging: From Physiology to Therapy, FRE 3313 CNRS/Université Bordeaux 2, 146 rue Léo Saignat, F - 33076 Bordeaux, France

2: Hôpital Cardiologique du Haut-Lévèque, Bordeaux, France

3: Philips Healthcare, France

Correspondence: Baudouin DENIS de SENNEVILLE

Laboratory for Molecular and Functional Imaging: From Physiology to Therapy, FRE 3313 CNRS/Université « Victor Segalen » Bordeaux 2

146, rue Léo Saignat, case 117, 33076 Bordeaux, France

E-Mail: baudouin@imf.u-bordeaux2.fr

Tel: +33 5575745 91, Fax: +33 557574597

Word count: 3733 
Short title: Feasibility of fast MR-thermometry during cardiac RF ablation

\section{Abstract}

Background: Online MR temperature monitoring during radiofrequency (RF) ablation of cardiac arrhythmias may improve the efficacy and safety of the treatment.

Methods: MR thermometry at 1.5 Tesla with the Proton Resonance Frequency method was assessed in 10 healthy volunteers under normal breathing conditions, using a multi-slice, ECG-gated, Echo Planar Imaging sequence in combination with slice tracking. Temperature images were post-processed to remove residual motion-related artifacts. Using an MR-compatible steerable catheter and electromagnetic noise filter, RF ablation was performed in the ventricles of two sheep in vivo. The standard deviation of the temperature evolution in time (TSD) was computed.

Results: Temperature mapping of the left ventricle was achieved at an update rate of approximately 1 Hz with mean TSD $3.6 \pm 0.9^{\circ} \mathrm{C}$. TSD measurements at the septum showed a higher precision $(2.8 \pm 0.9$ $\left.{ }^{\circ} \mathrm{C}\right)$ than at myocardial regions at the heart-lung and heart-liver interfaces $\left(4.1 \pm 0.9^{\circ} \mathrm{C}\right)$. Temperature rose maximally by $9{ }^{\circ} \mathrm{C}$ and $16{ }^{\circ} \mathrm{C}$ during $5 \mathrm{~W}$ and $10 \mathrm{~W}$ RF applications respectively for 60 seconds each.

Conclusion: Tissue temperature can be monitored at an update rate of approximately $1 \mathrm{~Hz}$ in five slices. Typical temperature changes observed during clinical RF application can be monitored with an acceptable level of precision.

Key words: MR thermometry, cardiac arrhythmia, ablation. 


\section{Abbreviations:}

\begin{tabular}{|c|c|}
\hline $\mathrm{RF}$ & - Radiofrequency \\
\hline $\mathrm{AF}$ & - Atrial fibrillation \\
\hline VT & - Ventricular tachycardia \\
\hline FOV & - Field of View (FOV) \\
\hline $\mathrm{TE}$ & - Echo Time \\
\hline TR & - Repetition Time \\
\hline EPI & - Echo Planar Imaging \\
\hline SENSE & - Sensitivity Encoding \\
\hline AV & - Atrioventricular \\
\hline IV & - Intravenous \\
\hline PRF & - Proton Resonance Frequency \\
\hline SNR & - Signal-To-Noise ratio \\
\hline $\mathrm{SD}$ & - Standard deviation \\
\hline TSD & - Temperature standard deviation \\
\hline ROI & - Region of interest \\
\hline BW & - Box and Whiskers \\
\hline
\end{tabular}




\section{Introduction}

Since 1987, when the radiofrequency (RF) energy was first used in the ablation of atrioventricular (AV) node in dogs, this form of energy has been the predominant means of treating cardiac arrhythmias in the catheterization laboratory $(1,2)$. Amongst various forms of energy, RF energy is safe, reproducible and can create discrete and permanent lesion in a controlled manner $(3,4)$. Despite the advent of irrigated-tip catheters, RF energy is limited in its ability to deliver deep lesions with adequate safety margin $(5,6)$. Catheter ablation of accessory pathways and atrio-ventricular nodal re-entry is facilitated by superficial and focal nature of the target tissue. However, deep and transmural lesions are frequently not feasible with endocardial and/or epicardial RF ablation of arrhythmias like atrial fibrillation and ventricular tachycardia which have complex and extensive substrates. Although, monitoring temperature at the electrode tip using a thermocouple and internal/external cooling of the ablation electrode has increased the safety and efficacy of the treatment, tissue temperature remains largely undetermined. Notably, the discrepancy between the electrode-tip temperature and tissue temperature can be $>30^{\circ} \mathrm{C}$, particularly with irrigated-tip catheters. This is a major issue as the lesion size is directly linked to tissue temperature. Knowing the tissue temperature during RF delivery could help to deliver the optimal magnitude of energy to achieve transmural and stable lesions without the risks of steam pops, perforation and damage to collateral extracardiac structures. In addition, the risk of arrhythmia recurrence for the most complex and widely prevalent arrhythmias like atrial fibrillation (AF) and ventricular tachycardia (VT) can be minimized if transmural and permanent lesions can be applied.

MR thermography is a novel, non-invasive, tissue-temperature monitoring tool. Clinical feasibility has been demonstrated for mobile organs such as liver and kidneys $(7,8)$. Recent data showed that MR thermometry of the heart was feasible with ECG and respiratory triggering but with 
limited temporal resolution, making precise dosimetry of the deposited energy difficult (9). We hypothesized that MRI could be used for tissue-temperature monitoring of the heart at an update rate of the cardiac cycle.

\section{Material \& Method}

Human study:

A preliminary study was performed in healthy human volunteers $(N=10)$ using Philips Achieva 1.5 Tesla (Philips Healthcare, Best, The Netherlands) MRI. A cardiac coil with three rigid elements located on the bottom and 2 flexible elements on the top was used for image acquisition. Electro cardiogram signals were recorded continuously with an MR-compatible system including 4 leads surface electrodes. After acquiring scout views (Figure 1), the short axis of the heart was determined on true Fast Imaging with Steady state Precession (true-FISP) images (10). This geometry was used to investigate the precision of thermometry in the left ventricular myocardium. The acquisition sequence was triggered by the cardiac signal and one data set was acquired per cycle. Six contiguous slices were acquired sequentially within a single heart beat with the following parameters to obtain a volume of interest: $250 \times 166 \mathrm{~mm}$ rectangular Field of View (FOV), 96×64 matrix (resulting in an in-plane resolution of $2.6 \mathrm{~mm}$ for an acquisition time of $37 \mathrm{~ms}$ per slice), $7 \mathrm{~mm}$ slice thickness, $20 \mathrm{~ms}$ Echo Time (TE), Repetition Time (TR) equal to the period of one cardiac cycle and single shot Echo Planar Imaging (EPI) with 1.5 acceleration factor using parallel imaging with Sensitivity Encoding (SENSE) resulting in an effective echo train of 43 echoes (11). Reconstructed images were zero-filled to $112 \times 112$ pixels. Saturation slabs were positioned on each side of the imaging stack to reduce the 
intensity of the blood signal on the MR images. The slice location was dynamically adjusted using a pencil-beam navigator positioned at the liver/lung interface (slice tracking technique) in order to compensate for respiratory motion. A factor of 0.6 (default value of the MR scanner) in the amplitude of the measured displacement of the diaphragm was applied for repositioning the MR-imaging stack in short-axis. The triggering delay was adjusted to acquire the last slice in the mid- or end-diastole. No arrhythmia rejection criteria or slice tracking limits were applied to ensure a systematic acquisition of a complete stack independent of potential jitter in the period of cardiac cycle and/or variation in respiratory amplitude. In each volunteer, the volume was continuously acquired 200 times over a period of 3 to 5 minutes depending on the individual's heart rate.

\section{Animal study:}

Animal preparation for MR-guided RF-ablation: The experimental protocol was in compliance with the institutional rules for animal care and the French law on animal experimentation. Each animal $(N=2)$ weighing $55+/-4 \mathrm{~kg}$ was sedated by intramuscular injection of $0.1 \mathrm{mg} / \mathrm{kg}$ of acepromazine (Calmivet, Vetoquinol, Lure, France) and anesthetized by intravenous (IV) injection of $0.1 \mathrm{~mL} / \mathrm{kg}$ of pentobarbital (Ceva santé animale, Libourne, France). The general anesthesia was maintained by continuous IV sodium pentobarbital $(6 \mathrm{mg} / \mathrm{kg} / \mathrm{h})$ infusion. The animal was ventilated with $40 \%$ humidified oxygen via tracheostomy. The right femoral artery was cannulated with an 8 FR sheath. The MRI compatible RF-ablation catheter was advanced retrogradely and positioned into the left ventricle under fluoroscopic guidance. The animal was moved to the MR Lab for temperature imaging. After completion of the experiment, the animal received a lethal IV dose of Dolethal.

MR-compatible RF-ablation device: A 7 French MR-compatible, non-perfused, steerable catheter (Bard, Electrophysiology Division, MA USA), equipped with a single electrode tip for RF-ablation was 
used . The reference electrode was positioned on the posterior leg of the animal ensuring good skin contact. The catheter and reference electrodes were connected to RF generator (AG 1006, Germany) located outside the Faraday cage. The electrical output $(500 \mathrm{kHz})$ of the generator was filtered by a series of 4 notch passive filters (inserted at the output of the RF generator, on each side of the Faraday cage and near the electrical plug of the catheter) tuned to the MR resonant frequency (64 MHz) in order to suppress the electromagnetic interference with the quality of MR images. RF catheter was not capable of recording any electrical signal or tip temperature. The susceptibility artifact induced by the catheter tip was evaluated on an $\mathrm{Agar}$ gel doped with $\mathrm{NaCl}$ to simulate the electrical impedance of living tissues.

MR-acquisition protocol: MRI guided RF ablation was undertaken in vivo in the left ventricle of the sheep. Real-time MR thermometry was performed during RF ablation using the acquisition protocol of the human study including ECG-triggered and respiratory-compensated acquisition of 400 dynamic images of cardiac short axis. Five slices were acquired per heart beat with navigator based slice tracking using the following parameters: $\mathrm{TR} / \mathrm{TE}=650 / 16 \mathrm{~ms}$, bandwidth in readout direction $=2085 \mathrm{~Hz}$, flip angle $=35^{\circ}, \mathrm{FOV}=320 \times 140 \mathrm{~mm}^{2}$, slice thickness $=6 \mathrm{~mm}$ and matrix $=128 \times 56$.

\section{Data processing:}

Processing of thermal maps: Despite introducing compensations for cardiac and respiratory motions, variations could occur in the duration of cardiac cycle during image acquisition over the examination period. In addition, slice tracking was performed in the slice direction, without in-plane repositioning of the FOV. Therefore, combination of both effects required additional in-plane image registration (12). Moreover, phase changes induced by local susceptibility changes related to lung volume modifications and liver displacement $(13,14)$ needed to be compensated for in the time series of the phase images 
prior to temperature calculation with the Proton Resonance frequency (PRF) shift technique (Figure 2) $(15,16)$. To account for these effects, the image processing was performed in two steps viz. image registration for temporal analysis of temperature evolution, and compensation of background phase related to motion-induced susceptibility changes:

- Step 1: In-plane image registration. In-plane motion compensation was performed by analyzing the magnitude images (17). For this purpose, the first image in the time series was selected as a position reference. Assuming a global translation displacement, a gradient-driven descent algorithm maximizing the inter-correlation coefficient between the reference and the current image was initially performed on each image of the time series (18). The resulting estimated displacement was then used for preconditioning a more complex optical flow algorithm (originally proposed by Horn and Schunck (19)) for image registration on a pixel-bypixel basis. The algorithm included a modification proposed by Cornelius and Kanade (20) which relaxes the intensity conservation condition from global to regional conservation. In order to optimize the computation time and stabilize the convergence, a multi-resolution scheme was implemented which iterates up the registration from a four-fold down-sampled image to full image resolution (21).

- Step 2: Correction of susceptibility related phase changes with motion. The first images in the time series were stored in a multi-baseline collection, including registered phase images and the corresponding magnitude image $(22,23)$. Since the amplitude of cardiac displacement was lower for a mechanically ventilated animal than for an awake human, the stack of first 50 images acquired in the time series (referred to as "dynamics" in the text) was stored in the collection for animal experiments, whereas the stack of first 100 dynamics was used for volunteers. The subsequent dynamics were corrected with this reference data set. For this purpose, the current magnitude image was compared to each of the magnitude images of the collection by computing the list of inter-correlation coefficients. The phase-image of the 
collection corresponding to the maximal inter-correlation value within this list was subtracted to retrieve the susceptibility-corrected temperature map. The motion correction algorithm has been implemented in $\mathrm{C}$ and required approximately $100 \mathrm{~ms}$ of total computation time for one image with a resolution of $128 \times 128$ on a dual processor dual core AMD Opteron $2.4 \mathrm{GHz}$ with $8 \mathrm{~GB}$ of RAM, demonstrating that it is compatible for real-time processing.

Assessment of temperature stability: The stability of thermometry was evaluated in healthy volunteers without myocardial heating. Statistical evaluation of the temperature standard deviation was performed on each volunteer over a region of interest (ROI) covering the entire left ventricle myocardium. For each pixel, the standard deviation of the temperature evolution in time (TSD) was computed and the resulting image was analyzed. For this purpose, the mean value of the TSD in the selected ROI was calculated for all volunteers and the distribution of these values for each segment was plotted with a Box and Whiskers (BW) graphical representation. In this representation, 5 temperature levels corresponding to the first decile (T10), the first quartile (T25), the median (T50), the third quartile (T75) and the last decile (T90) of the distribution of the TSD values were reported. BW analysis was performed on the ROI covering the entire myocardium for each of the six segments defined by the standardized myocardial segmentation and nomenclature (24). The ROI delimiting each region was drawn by the same operator to avoid interoperator variability.

\section{Results}

\section{Human study:}

The durations of respiratory and cardiac cycles ranged from 6 to $8 \mathrm{sec}$ and 0.8 to $1.5 \mathrm{sec}$, 
respectively. Representative MR images of the thermometry sequence at two extreme positions in the respiratory cycle are displayed in Figure 2. The Signal-To-Noise ratio (SNR) of the magnitude image of the left ventricular myocardium (mean $-19.8 \pm 6$, range 14 to 32 ) was sufficient in all participants to apply the motion correction algorithm. The local phase variations associated with respiration-induced susceptibility changes were observed during respiratory cycle (see Figures 2.c and 2.d).

The TSD maps obtained with and without the multi-baseline correction in the worst case are shown in Figures 3.a and 3.b, respectively. The temperature evolution in a single pixel located in the septum displayed periodic oscillations associated with respiration, with uncorrected maximal amplitude of $50{ }^{\circ} \mathrm{C}$ (Figure 3c). After correction, the oscillations were reduced leading to a TSD value lower than $5{ }^{\circ} \mathrm{C}$ in $75 \%$ pixels included in a ROI covering the left ventricle. The maximal value obtained was 5.7 ${ }^{\circ} \mathrm{C}$.

The TSD reached several tens of degrees in absence of specific corrections in 10 volunteers. The use of multi-baseline correction method resulted in reduction of the TSD in all cases. The TSD of the left ventricle was $3.6 \pm 0.9^{\circ} \mathrm{C}$ (median $-3.7^{\circ} \mathrm{C}$, range $2.5-5.4{ }^{\circ} \mathrm{C}$ ). T90 values of the distribution of the TSD (Figure 4a) ranged from $3.4^{\circ} \mathrm{C}$ (volunteer \#4) to $6.3{ }^{\circ} \mathrm{C}$ (volunteer \#9), with a mean of $4.8 \pm 1$ ${ }^{\circ} \mathrm{C}$. The $\mathrm{T} 75$ values ranged from $2.7^{\circ} \mathrm{C}$ to $5^{\circ} \mathrm{C}$ (mean $-3.8 \pm 0.8^{\circ} \mathrm{C}$ ). The $\mathrm{T} 50$ values ranged from 2.1 ${ }^{\circ} \mathrm{C}$ to $2.9{ }^{\circ} \mathrm{C}$ for 5 volunteers $(\# 1, \# 2, \# 3, \# 4, \# 5)$ and from $4{ }^{\circ} \mathrm{C}$ to $4.6{ }^{\circ} \mathrm{C}$ for volunteers $\# 6$ and \#9. For the remaining 3 volunteers $(\# 7, \# 8 \# 10)$, it was $3.3{ }^{\circ} \mathrm{C}$. The T10 values were below $3{ }^{\circ} \mathrm{C}$ for $8 / 10(80 \%)$ volunteers and between 3 and $4{ }^{\circ} \mathrm{C}$ for volunteers \#6 and \#9. The analysis of the TSD for each segment integrating all the volunteers, revealed similar distributions for segments 1, 2, 3 and 6, with T50 values around $3{ }^{\circ} \mathrm{C}$ and T75 values below $3.5{ }^{\circ} \mathrm{C}$ (Figure 4b). The T50 values of segments 4 and 5 were approximately $4{ }^{\circ} \mathrm{C}$ each, and $\mathrm{T} 75$ values ranged from $4.3{ }^{\circ} \mathrm{C}$ to $5^{\circ} \mathrm{C}$. The average value of the TSD for segments $1,2,3$ and 6 was $3{ }^{\circ} \mathrm{C}$ whereas it rose to $4.1{ }^{\circ} \mathrm{C}$ for segments 4 and 5 . 
A hypo-intense signal ( $3 \mathrm{~mm}$ in size) was observed on the magnitude image of the thermometry sequence around the catheter tip. Negligible loss of image quality was observed during RF-ablation on temperature images, indicative of efficient filtering of electromagnetic interferences.

In-vivo RF-ablation of the left ventricle in sheep:

The duration of cardiac cycle was approximately $650 \mathrm{~ms}$. The SNR on the magnitude image was 20 and TSD prior to $\mathrm{RF}$ ablation was $1{ }^{\circ} \mathrm{C}$ on the whole myocardium. The latter rose to $2{ }^{\circ} \mathrm{C}$ at the interface between the tissue and the RF-ablation electrode (Fig 5a and 5d). MR-guided RF-ablation of the left myocardium showed consistent evolution of the tissue temperature, with a progressive increase near the catheter tip during $60 \mathrm{sec}$ energy delivery, followed by spontaneous tissue cooling by heat conduction and perfusion. The maximum increase in temperature in the pixel at the contact with the catheter tip for $5 \mathrm{~W}$ and $10 \mathrm{~W}$ RF delivery were $9{ }^{\circ} \mathrm{C}$ (Fig 5b) and $16{ }^{\circ} \mathrm{C}$ (Fig 5e), respectively. Temperature images acquired at the end of RF energy delivery showed larger area of heated tissue with $10 \mathrm{~W}$ RF power than $5 \mathrm{~W}$ (Fig $5 \mathrm{c}$ and $5 \mathrm{f}$ ). No heating was observed in the adjacent slices. Temperature elevations higher than $5{ }^{\circ} \mathrm{C}$ were achieved in ellipsoidal regions of dimension $7 \times 17 \mathrm{~mm}^{2}(5 \mathrm{~W} \mathrm{RF})$ and $10 \times 20 \mathrm{~mm}^{2}(10 \mathrm{~W} \mathrm{RF})$.

\section{Discussion}

This work demonstrates the feasibility of online cardiac tissue temperature monitoring during RF ablation with an MRI-compatible catheter. Compared to recently published data (9), our study accomplished improved temporal resolution and volume coverage, since 5-6 slices were acquired per 
cardiac cycle ( $\sim 1 \mathrm{sec}$ duration) instead of a single slice every 10 to $20 \mathrm{sec}$. Typical RF ablation duration is about $1 \mathrm{~min}$ per application. Therefore, acquiring only 3 to 6 temperature images during the ablation process may appear insufficient to characterize the temperature evolution and can render the evaluation of the thermal dose difficult. To overcome this major limitation, we proposed to accelerate the acquisition sequence using EPI in combination with ECG triggering, navigator based slice tracking and image processing for compensation of motion-related susceptibility artifacts. In animal experiments, the RF-ablation catheter was observed on the slice of the time series, indicative of an efficient slice repositioning of the imaging stack from navigator data acquired on the diaphragm. In the present study, preference was given to the speed of acquisition. Single-shot acquisition of several slices per cardiac cycle was obtained. Other acquisition strategies such as multi-shot EPI or PROPELLER acquisition techniques (25) splitted over several successive cardiac cycles may be investigated in order to increase the spatial resolution which may come at the cost of the update rate of MR-thermometry. However, in case of variations in the cardiac cycle duration, the resultant image may be corrupted by important artifacts. The balance between acquisition speed and temporal resolution will depend on the SNR of the resultant MR temperature images, which may vary as a function of the patient anatomy (size of the thorax), the performance of the MR receiver coil (number of coil elements, geometry and spatial arrangement), and the RF-ablation catheter characteristics (irrigated vs. non-irrigated).

This method reduces the complex 3D motion of the heart to a 2D in-plane motion, for which inplane image registration algorithms can be applied. Although the left ventricle mainly displayed a translational displacement, the estimation of a complex deformation with sub-pixel precision on a voxel-by-voxel basis was necessary. The algorithm implemented for image registration was well-suited for compensation of the left ventricular displacements after the motion-effect was reduced by cardiac synchronization and the slice position dynamically adjusted by the navigator. When the true motion field violates the brightness consistency model used for its approximation, it predominantly limits the image-registration based on the image intensity. Indeed, any temporal change in the image intensity 
was attributed to motion. In this study, the blood signal was suppressed, since the blood-flow artifacts make the estimation of complex local myocardial deformation difficult. However, during heating process (hyperthermia), several MR relevant tissue properties such as $T_{1}$ and $T_{2}$ relaxation times may change and lead to local signal intensity variations in the heated region. Therefore, these signal intensity variations may be interpreted as local motions in the registration process. In the present study, energy delivery was limited by absence of an irrigated-tip ablation catheter. Hence, relatively low power RF energy could be applied $\left(\max =10 \mathrm{~W}\right.$ ), leading to a maximal temperature elevation of $16{ }^{\circ} \mathrm{C}$. Thus, the possibility of incorrect registration is expected to be negligible. It should also be noted that the presence of saturation slabs positioned on each side of the imaging stack (Figure 1) may induce appearance and disappearance of surrounding tissue in the image field which may disturb the registration process based on conservation of local image intensities.

Despite ECG triggering to counter the influence of cardiac motion, a multi-baseline correction was required to correct susceptibility-related phase changes induced by periodic cardiac displacement with respiration. Since phase variations associated with motion were observed during the preceding learning phase and subsequently applied to correct the MR-thermometry during intervention, the method could not intrinsically correct MR-thermometry artifacts associated with spontaneous motion. If new positions are observed during hyperthermia, a recalibration of the phase correction data is required, which is a disadvantage when compared with other approaches such as reference-less correction $(26,27)$. However, compared to reference-less correction methods, the proposed multibaseline correction allows for an accurate correction of susceptibility-related phase changes even in the regions with complex susceptibility distributions or signal discontinuities such as the heart-liver interface (regions 4 and 5).

Temperature monitoring of the left ventricle was feasible with an acceptable level of precision (median TSD approximately $3{ }^{\circ} \mathrm{C}$, except on segments 4 and 5). Stability was better in the septum (regions 2 and 3) and the regions 1 and 6. In comparison with the others, the regions 4 and 5 which 
depicted a higher TSD were more prone to spatial and temporal susceptibility variations responsible for susceptibility artifacts because they were located at the liver/heart/lung interface. Since the lower precision boundary of PRF-based MR-thermometry on static targets is directly proportional to the inverse of the SNR of the employed sequence (28), it is possible to evaluate the effectiveness of the correction strategies for dynamic phase artifacts by direct comparison. In humans, the SNR study led to a minimal achievable TSD of $1.1 \pm 0.3{ }^{\circ} \mathrm{C}$ (range 0.6 to $1.4{ }^{\circ} \mathrm{C}$ ). In animal experiment, it was inching closer to the theoretical values when the multi-baseline correction was used but residual motion artifacts and image distortions inherent to EPI acquisitions prevented it from getting better.

The TSD in each analyzed region in the volunteers was approximately $50 \%$ higher than those reported in the mechanically ventilated animals by Kolandaivelu (9). However, at an update rate at least 10 times higher, it was acceptable for efficient monitoring of temperature evolution in the myocardium. The precision of the estimated temperature and the resulting accumulated thermal dose could be improved by temporal filtering of the temperature curves in each pixel with a low pass filter, as suggested by Roujol (17).

During $\mathrm{RF}$ ablation, the precision of the estimated temperature was better in animals than humans by a factor of $\sim 2$. This could be attributed to the well-controlled breathing conditions in animals which lowered the amplitude and speed of cardiac displacement along the caudo-cranial axis, reducing the risks of uncompensated out-of-plane motion associated with imperfect slice tracking. Fastand motion-compensated thermometry allowed visualization of the temperature evolution with a temporal resolution less than $1 \mathrm{sec}$. RF catheter generated minimal artifact on the temperature images and the rise in temperature was consistent with RF energy deposition.

Future perspectives of this work which is focused on developing a rapid temperature-imaging methodology should include the evaluation of the accuracy of thermometry in presence of cardiac arrhythmias where jitter in the cycle duration is associated with unobserved contraction patterns during 
the learning phase. Therefore, further developments in data processing may be necessary to manage images efficiently. In addition, the voxel size of the thermometry sequence remains too large to precisely characterize small lesions created by low-energy RF delivery. Thus, despite encouraging results obtained with the current technology which has been developed using non-irrigated RF catheter, improvements are necessary when an irrigated-tip-catheter is used for creating transmural lesions. This technique would also pave way for establishing correlation between the thermal dose calculated from the temperature images and the post-ablation DE-MRI (9) and/or the histological analysis of RF lesion. Other technological aspects like active slice tracking based on a sensor embedded in the catheter and/or imaging with the catheter tip can be considered (29). Finally, improved spatial resolution imaging is required to improve the image quality in the atrium during $\mathrm{AF}$ ablation.

\section{Conclusion}

MR thermometry of the human heart is feasible at an update rate of 5 images/cardiac cycle with a precision of $3.6{ }^{\circ} \mathrm{C} \pm 0.9(\min =2.5, \max =5.4)$. Combination of slice tracking, ECG triggering, efficient image registration and modeling of susceptibility changes related to motion allows for significant improvements in the precision of thermometry of the human heart wherein cardiac cycle provides the temporal resolution. The standard deviation of the tissue temperature is acceptable in the setting of local temperature increases achieved during catheter ablation. In animals the preliminary results demonstrate the feasibility of real-time tissue temperature monitoring during RF delivery.

\section{Acknowledgements}


Ligue Nationale Contre le Cancer, Conseil Régional d'Aquitaine, Diagnostic Molecular Imaging ECFP6-project LSHB-CT-2005-512146 "Agence National de Recherche (project MRgHIFU-ALKT)", Fondation InNaBioSanté (project ULTRAFIT)", Philips Medical System. Dr Ashok Shah is greatfully acknowledged for english editing of the manuscript.

\section{References}

[1] Chugh A, Morady F. Atrial fibrillation: Catheter ablation. J. Interv. Card. Electrophysiol. 2006; $16: 15-26$.

[2] Jaïs P, Cauchemez B, Macle L, Daoud E, Khairy P, Subbiah R, Hocini M, Extramiana F, Sacher F, Bordacher P, et al. Catheter ablation versus antiarrhythmic drugs for atrial fibrillation: The A4 study. Circulation 2008; 118(24):2498-2505.

[3] Malchano Z, Neuzil P, Cury R, Holmvang G, Weichet J, Schmidt E, Ruskin J, Reddy V. Integration of cardiac CT/MR imaging with three-dimensional electroanatomical mapping to guide catheter manipulation in the left atrium: Implications for catheter ablation of atrial fibrillation. J. Cardiovasc. Electrophysiol. 2006; 17:1221-1229.

[4] Saremi F, Tafti M. The role of computed tomography and magnetic resonance imaging in ablation procedures for treatment of atrial fibrillation. Semin. Ultrasound CT MR 2009; 30(2):125-156.

[5] Wittkampf FH, Nakagawa H. Rf catheter ablation: Lessons on lesions. Pacing Clin Electrophysiol. $2006 ; 29: 1285-1297$. 
[6] Ouyang F, Antz M, Ernst S, Hachiya H, Mavrakis H, Deger FT, Schaumann A, Chun J, Falk P, Hennig D, Liu X, Bansch D, Kuck KH. Recovered pulmonary vein conduction as a dominant factor for recurrent atrial tachyarrhythmias after complete circular isolation of the pulmonary veins: Lessons from double lasso technique. Circulation. 2005; 111:127-135.

[7] Isihara Y, Calderon A, Watanabe H, Okamoto K, Suzuki Y, Kuroda K, Suzuki Y. A precise and fast temperature mapping using water proton chemical shift. Magn. Res. Med. 1995; 34(6):814-23.

[8] Seror, Lepetit-Coiffé M, Bail BL, de Senneville BD, Trillaud H, Moonen C, Quesson B. Real time monitoring of radiofrequency ablation based on MR thermometry and thermal dose in the pig liver in vivo.

[9] Kolandaivelu A., Zviman M. M., Castro V., Lardo A. C., Berger R. D., Halperin H. R., Noninvasive Assessment of Tissue Heating During Cardiac Radiofrequency Ablation Using MRI Thermography, Circulation: Arrhythmia and Electrophysiology, 2010;3(5):521-529.

[10] Deimling M, Heid O. Magnetization prepared true FISP imaging. Proceedings of the 2nd Annual Meeting of ISMRM, San Francisco, CA, USA, 1994 (Abstract 495).

[11] Pruessmann KP, Weiger M, Scheidegger MB, Boesiger P. SENSE: sensitivity encoding for fast MRI. Magn Reson Med 1999;42:952-962.

[12] Maintz J. B. A., Viergever M. A., A survey of medical image registration. Medical Image Analysis $1998 ; 2: 1-36$. 
[13] Young I. R., Hajnal J. V., Roberts I. G., Ling J. X., Hill-Cottingham R. J., Oatridge A., Wilson J. A., An evaluation of the effects of susceptibility changes on the water chemical shift method of temperature measurement in human peripheral muscle, Magnetic Resonance in Medicine, 1996, 36(3):366-74.

[14] Peters R. D., Henkelman R. M., Proton-resonance frequency shift MR thermometry is affected by changes in the electrical conductivity of tissue, Magnetic Resonance in Medicine, 2000, 43:62-71.

[15] Quesson B., de Zwart, J.A., Moonen C. T. W., Magnetic resonance temperature imaging for guidance of thermotherapy. J Magn Reson Imaging. 2000 Oct;12(4):525-33.

[16] Rieke V., Butts Pauly K. MR thermometry. J Magn Reson Imaging. 2008; 27:376-390.

[17] Roujol S., Ries M., Quesson B., Moonen C. T. W., Ries M., Denis de Senneville B., Real-time MR-Thermometry and Dosimetry for interventional guidance on abdominal organs, Magnetic Resonance in Medicine, 2010, Volume 63 Issue 4, Pages 1080 - 1087.

[18] Press W., Teukolsky S., Vetterling W., Flannery B., Numerical Recipes in C, their third edition, The Art of Scientific Computing, ISBN 0-521-43108-5. Cambridge University Press.

[19] Schunck B.G. Horn K.P. - Determining optical flow. Artificial intelligence 1981; 17:pp. 185-203.

[20] Cornelius N., Kanade T., Adapting optical flow to measure object motion in reflectance and XRay image sequences, ACM SIGGRAPH/SIGART Interdisciplinary Workshop on motion: 
representation and perception, Toronto, Canada, April 83.

[21] Pratikakis I., Barillot C., Hellier P., Mémin E., Robust multiscale deformable registration of 3D ultrasound images. International Journal of Image and Graphics, 3(4):547-566, 2003.

[22] Vigen K. K., Daniel B. L., Pauly J. M., Butts K., Triggered, navigated, multi-baseline method for proton resonance frequency temperature mapping with respiratory motion, Magnetic Resonance in Medicine, 2003, 50(5):1003-10.

[23] Denis de Senneville B., Mougenot C., Real time adaptive methods for treatment of mobile organs by MRI controlled High Intensity Focused Ultrasound, Magnetic Resonance in Medicine, 2007 Feb, $57(2): 319-30$.

[24] Cerqueira M. D., Weissman N. J., Dilsizian V., Jacobs A. K., Kaul S., Laskey W. K., Pennell D. J., Rumberger J. A., Ryan T., Verani M. S., American Heart Association Writing Group on Myocardial Segmentation and Registration for Cardiac Imaging, Standardized myocardial segmentation and nomenclature for tomographic imaging of the heart: a statement for healthcare professionals from the Cardiac Imaging Committee of the Council on Clinical Cardiology of the American Heart Association, Circulation.2002 Jan 29;105(4):539-42.

[25] Pipe, J.G. et al., Multishot Diffusion-Weighted FSE Using PROPELLER MRI, Magnetic Resonance in Medicine, 2002, vol. 47, pp. 42-52.

[26] Holbrook A. B., Santos J. M., Kaye E., Rieke V., Butts Pauly K., Real-time MR thermometry for monitoring HIFU ablations of the liver, Magnetic Resonance in Medicine. 2009, Vol. 63 Issue 2, p365- 
373.

[27] Grissom W. A., Holbrook A. B., Rieke V. A., Lustig M., Santos J. A., Swaminathan A., McConnell M. V., Butts Pauly K., Hybrid Multi-Baseline and Referenceless PRF-Shift Thermometry, Proceedings to the $18^{\text {th }}$ meeting of the International Society for Magnetic Resonance in Medicine, 2010, Stockholm.

[28] Conturo T. E., Smith G. D., Signal to Noise in Phase Angle Reconstruction : Dynamic Range Extension Using Phase Reference Offsets, Mag Res Med 15, 420-437. 1990.

[29] Peters D. C, Lederman R. J., Dick A. J., Raman V. K., Guttman M. A.,Derbyshire J. A., McVeigh E. R., Undersampled Projection Reconstruction for Active Catheter Imaging With Adaptable Temporal Resolution and Catheter-Only Views,Magn Reson Med. 2003 February; 49(2): 216-222. 


\section{Figures legends}
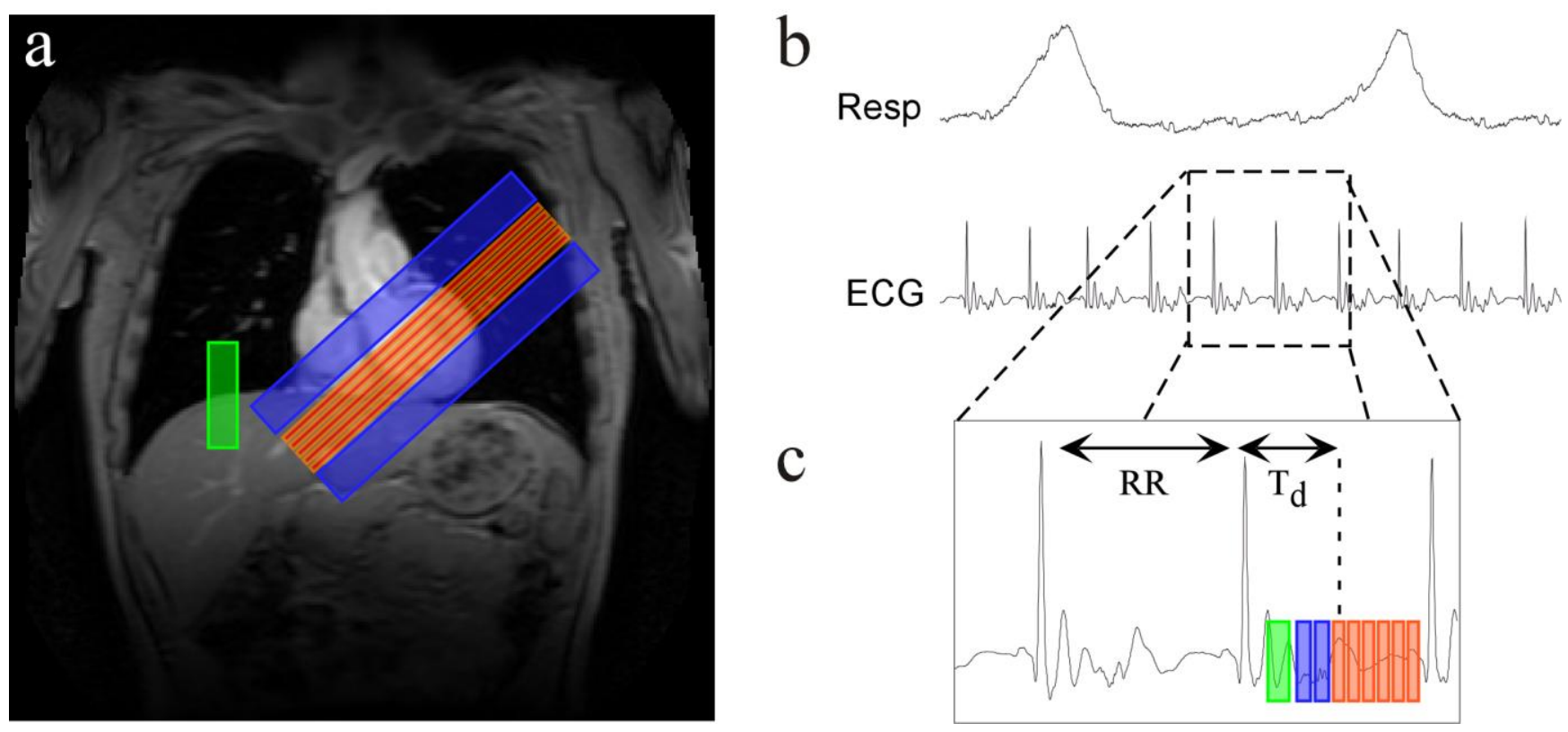

Figure 1. Experimental setup of temperature imaging of human left ventricle in short axis a: Coronal MR image. Slices used for temperature imaging are displayed in red. Slice position is adjusted online using a navigator positioned on the liver/lung interface (shown in green). Saturation slabs positioned on each side of the imaging stack are shown in blue.

b: Online respiratory and electro-cardiogram signals.

c: The complete stack is acquired in the mid- or end-diastole. The volume was acquired over at least 2 minutes to evaluate the precision and stability of the thermometry sequence. RR represents the duration of one cardiac cycle and $\mathrm{T}_{\mathrm{d}}$ is the temporal delay between the maximal ECG potential and the acquisition of the central slice of the imaging stack. 

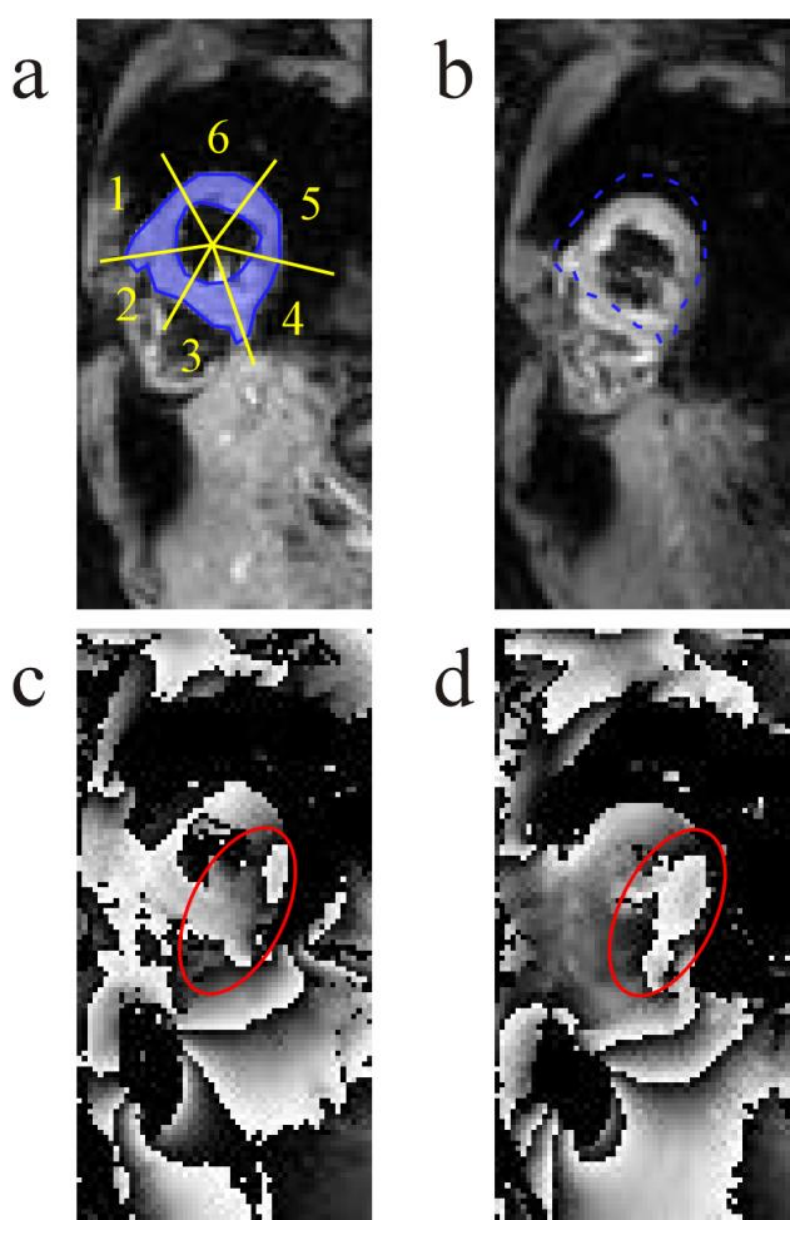

Figure 2. Example of MR images of the short axis of the human heart at two different instances during respiration.

a, b: anatomical images. a. Six segments defined by the standardized myocardial segmentation and nomenclature (24). The myocardial region of interest set manually for the temperature stability study is displayed in blue. The region of interest displayed in dashed line in $\mathbf{b}$ shows ventricular motion.

c, d: corresponding phase images. A region of interest manually set on the anatomical image to delimit the myocardium is displayed in solid blue line. Large phase changes induced by local susceptibility variations related to lung volume modifications and liver displacements can be predominantly seen at the heart/lung interface (red ellipse). 

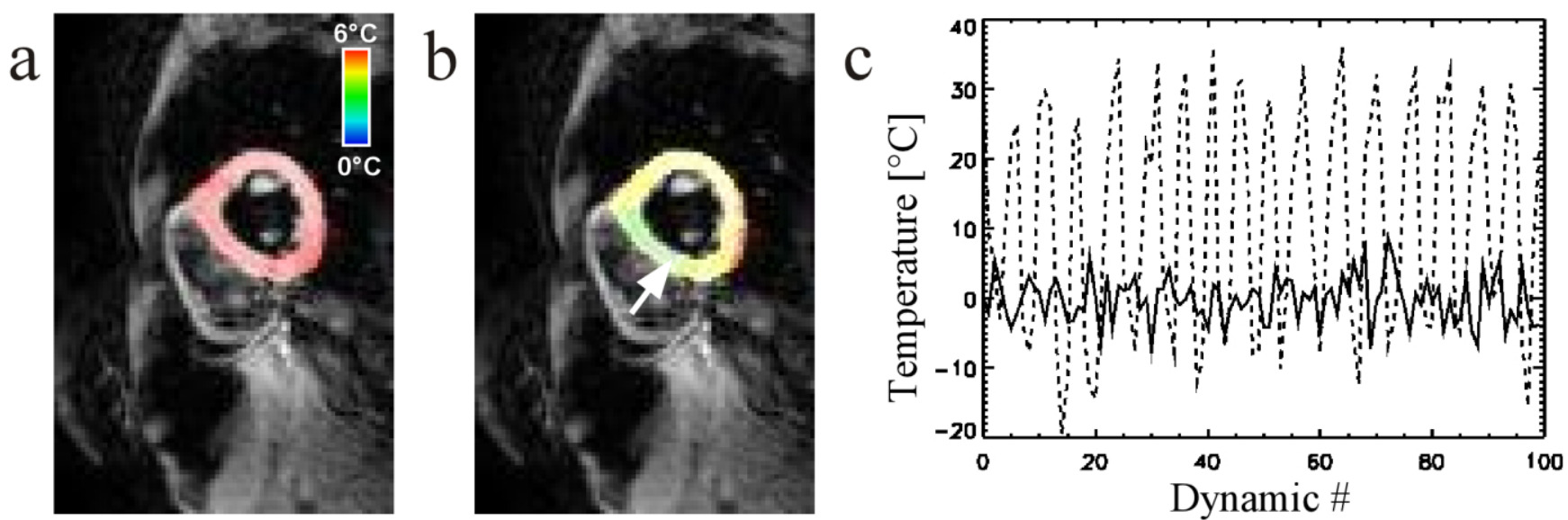

Figure 3. Worst temperature stability obtained on the left ventricle (volunteer \#6 in Figure 4). a: the temperature standard deviation map obtained without motion correction, b: the temperature standard deviation map obtained with motion correction, c: the temperature temporal evolution in a pixel located in the septum (white arrow on (b)) without (dashed line, $\mathrm{SD}=15.4{ }^{\circ} \mathrm{C}$ ) and with motion correction (solid line, $\mathrm{SD}=3.3^{\circ} \mathrm{C}$ ). 

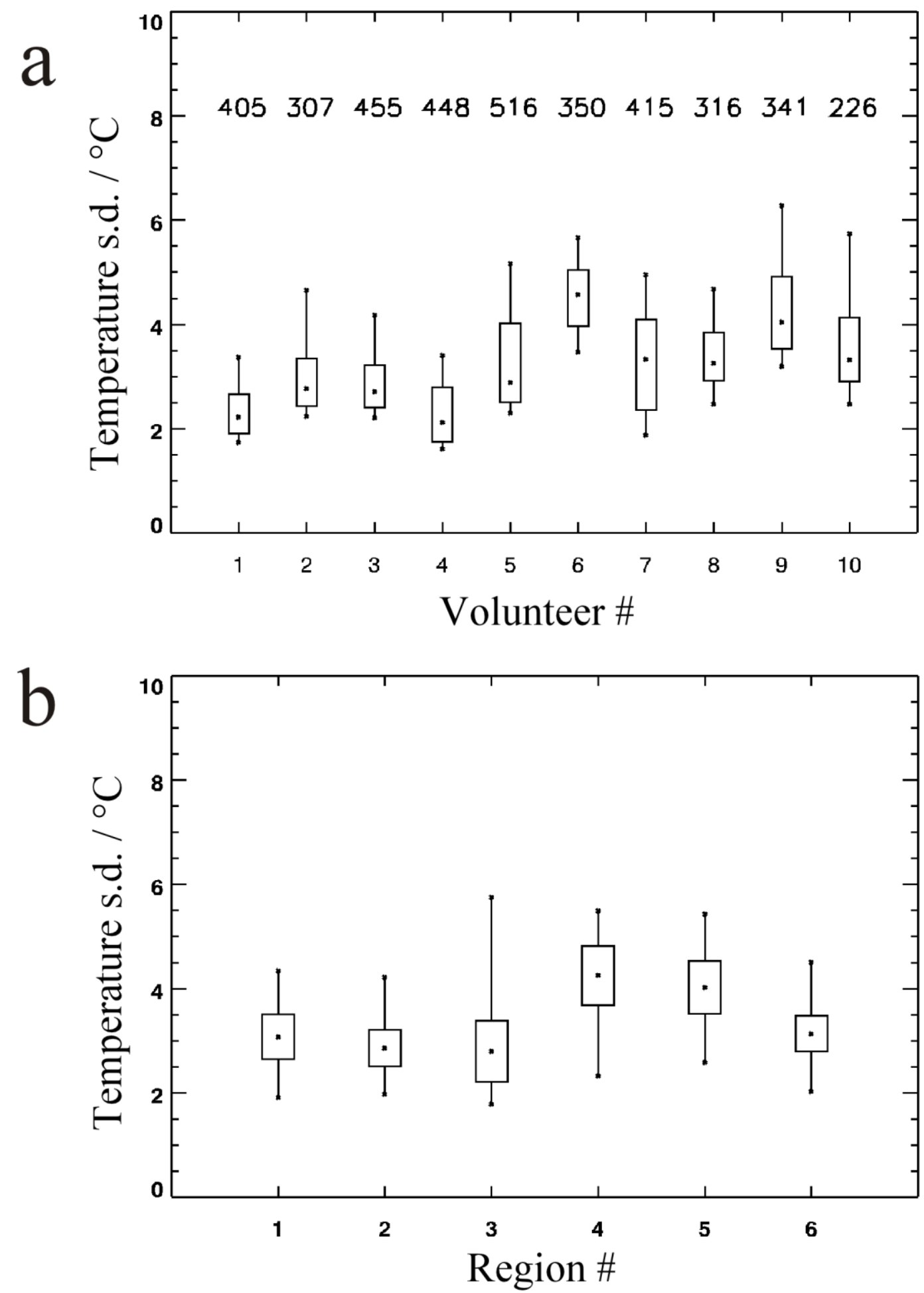

Figure 4. Temperature stability after compensation of motion-related susceptibility changes with the multi-baseline algorithm. a: Box and Whisker plot of the TSD of the left ventricular myocardium of each volunteer. For each volunteer, the plotted levels correspond to $10 \%$ (lowest point), first quartile (lower box limit), median (cross), third quartile (higher box limit) and 90\% (highest point) of the 
distribution of the TSD. The number of pixels in the region of interest is also shown. b: Box and Whisker plot of the TSD evaluated for each region of the standardized myocardial segmentation and nomenclature (24). Temperature levels correspond to the minimum (lowest point), the mean (cross), the standard deviation (box thickness) and the maximum (highest point) of the distribution of temperature standard deviation of all volunteers. 

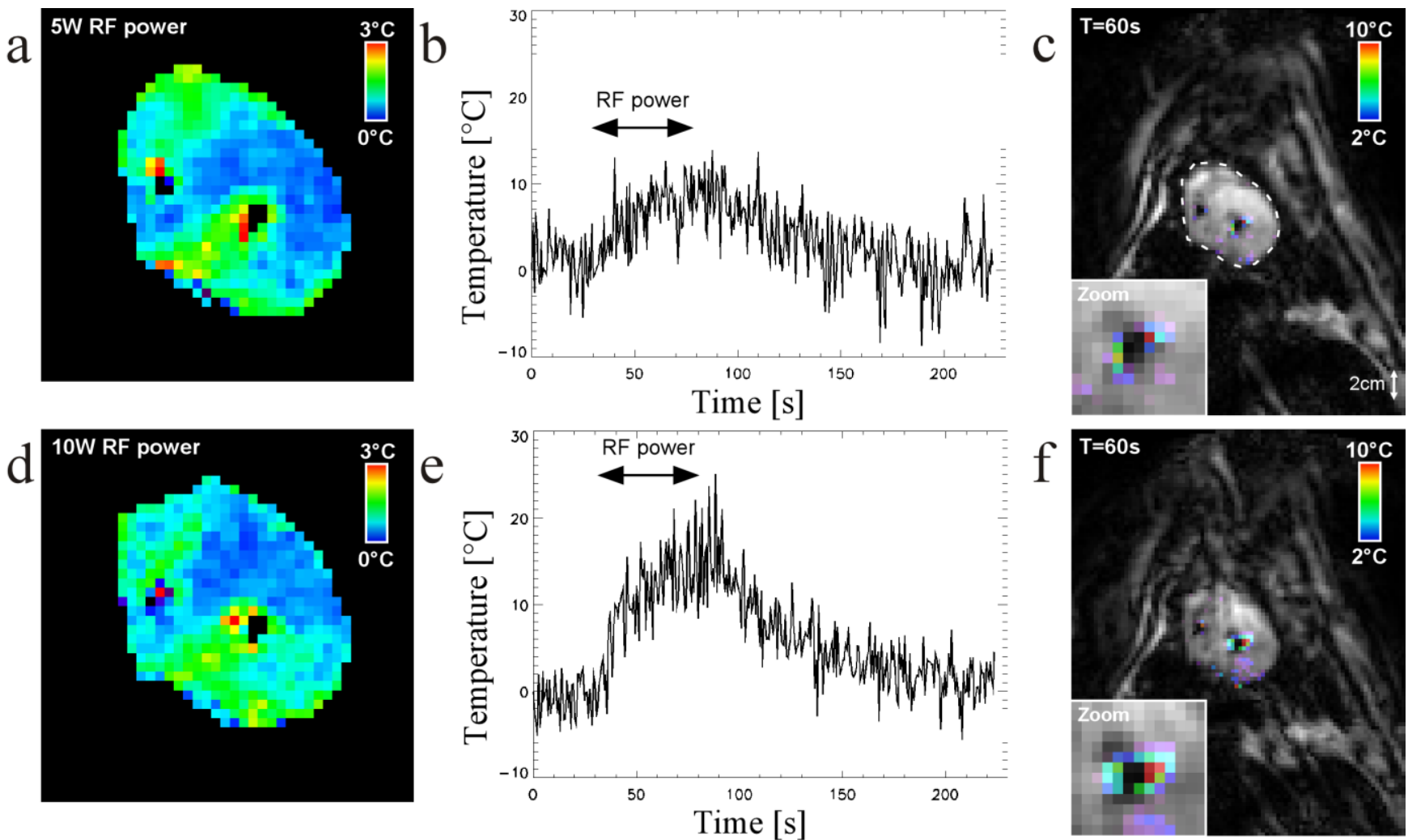

Figure 5. MR-Thermometry results of RF application on sheep's left ventricle.

a, d: TSD maps before hyperthermia during $5 \mathrm{~W}$ (a) and $10 \mathrm{~W}$ (d)RF applications. Temperature data are displayed in the ROI surrounding the heart (see c). b, e: temporal evolution of the temperature rise in the hottest pixel in the myocardium during $5 \mathrm{~W}$ (maximal temperature rise $-8^{\circ} \mathrm{C}$ ) (b) and $10 \mathrm{~W}$ (maximal temperature rise $\left.-16^{\circ} \mathrm{C}\right)(\mathrm{e}) \mathrm{RF}$ applications.

c, f: temperature distributions after one minute of $5 \mathrm{~W}$ (c) and $10 \mathrm{~W}$ (f) RF applications overlaid on the anatomical image. The myocardium is delimited by the white dashed line. 